\title{
A Power Aware Two Direction Error Correction and Detection Technique
}

\author{
Mahmoud Horr ${ }^{1}$, Houman Zarrabi ${ }^{2,}$ Hojjat Jafariarjmand ${ }^{3}$ \\ Faculty of Engineering, Science and Research branch of Azad University, Tehran, Iran ${ }^{1}$ \\ ICT Research Centre, Tehran, Iran $^{2}$ \\ Kar University, Tehran, $\operatorname{Iran}^{3}$
}

\begin{abstract}
This paper develops the error correction and detection method for Power aware error correction and detection and Increase the number of errors that can be detected and corrected in power state of digital communication devices. Consequence of this research can used in wireless sensors and IOT components because the prudence of power is important. This Method will dynamically change the method of error correction and detection based on power state of machine. Error correction and detection method that used is two dimensional error correction and detection based on hamming code. The circuits that create the redundancy bits and control of errors that maybe happened are simulated.
\end{abstract}

Keywords: Error correction and Detection, Two dimensional Hamming code, Dynamic power consumption.

\section{INTRODUCTION}

History of error correction and detection will be discussed. This paper study about a new two dimensional hamming error correction and detection. Redundant circuit for error correction of this method increase the power consumption of sender and receivers and is a problem in the sender and receiver circuits. It is important that we should decrease the power consumption in the confidence systems. In dynamic power aware systems it is possible to control amount of used power base on source power and make it better as a time function. Other method for error correction or detection used for example cyclic redundant check (CRC), checksum and gray codes are used in digital systems but mentioned methods can detect only but cannot to correct error bit(s).

\section{REVIEW OF LITERATURE}

The hamming cod was submitted by hamming in Bell Lab of United States at April 1945. Hamming increase k redundancies bit to $\mathrm{m}$ bit of message Based on equation (1). This equation is a general equation and for hamming code, that can correct only one error bit then $n$ is equal one and $\mathrm{K}$ is the number of redundant bits and $\mathrm{m}$ is number of data bits. Therefor the standard hamming code have $\mathrm{m}+\mathrm{k}$ bit at all. In this case the equation (2) was achieved.

$$
\sum_{i=0}^{n}\left(\begin{array}{c}
m+k \\
i
\end{array}\right) \leq 2^{k}
$$

Based on equation (2) the number of redundancy $\mathrm{k}$ bit is calculated. $\mathrm{K}$ bit is the minimum number of bits that must be added to data bits for one bit error correction be possible by hamming code hence distance of hamming will be 3 after increase the number of bits from $m$ to $m+k$ bits. This is shown in figure (1). By selecting $m=8$ then amount of $\mathrm{k}$ is 4 from equation (2).

$$
\sum_{\substack{i=0 \\
\mathrm{~m}+\mathrm{k}+1 \leq 2^{k}}}^{n}\left(\begin{array}{c}
m+k \\
i
\end{array}\right) \leq 2^{k} \Rightarrow \sum_{i=0}^{n=1}\left(\begin{array}{c}
m+k \\
i
\end{array}\right) \Rightarrow
$$

\begin{tabular}{|c|c|c|c|c|c|c|c|c|c|c|c|}
\hline $\mathrm{P}$ & $\mathrm{P}$ & $\mathrm{M}$ & $\mathrm{P}$ & $\mathrm{M}$ & $\mathrm{M}$ & $\mathrm{M}$ & $\mathrm{P}$ & $\mathrm{M}$ & $\mathrm{M}$ & $\mathrm{M}$ & $\mathrm{M}$ \\
1 & 2 & 3 & 4 & 5 & 6 & 7 & 8 & 9 & 1 & 1 & 1 \\
& & & & & & & & & 0 & 1 & 2 \\
\hline
\end{tabular}

Figure (1) -Hamming code structure $(m=8$ bits for message an $\mathrm{k}=4$ bits for redundancy)

In figure (1) the $p_{2^{i}}$ are redundancy and calculated by equation (3).

$$
\begin{gathered}
P 1=m 3 \oplus m 5 \oplus m 7 \oplus m 9 \oplus m 11 \\
P 2=m 3 \oplus m 6 \oplus m 7 \oplus m 10 \oplus m 11 \\
P 4=m 5 \oplus m 6 \oplus m 7 \oplus m 12 \\
P 8=m 9 \oplus m 10 \oplus m 11 \oplus m 12
\end{gathered}
$$

$\mathrm{Pi}$ is parity redundancy bit and $\mathrm{mi}$ is message bit in equation (3). And equation (4) used for error correction state that done in receiver.

$$
\begin{gathered}
C 1=P 1 \oplus m 3 \oplus m 5 \oplus m 7 \oplus m 9 \oplus m 11 \\
C 2=P 2 \oplus m 3 \oplus m 6 \oplus m 7 \oplus m 10 \oplus m 11 \\
C 4=P 4 \oplus m 5 \oplus m 6 \oplus m 7 \oplus m 12 \\
C 8=P 8 \oplus m 9 \oplus m 10 \oplus m 11 \oplus m 12
\end{gathered}
$$

It is possible that calculate error location bit by equation (5).

Error Location Index $=(8 * \mathrm{P} 8+4 * \mathrm{P} 4+2 * \mathrm{P} 2+\mathrm{P} 1)$

The error location bit is calculated by (5) equation. Error location calculate and hand in to decoder and index of error bit will export to error correction circuit and save to buffer. Error bit change after error location detected 
International Journal of Innovative Research in Electrical, Electronics, Instrumentation and Control Engineering

ISO 3297:2007 Certified

Vol. 5, Issue 2, February 2017

\section{III.TWO DIMENSIONAL ERROR DETECTION AND CORRECTION.}

We calculate the parity bits of hamming and move those bits to left corner of message bits that shown in figure (2).

$$
\begin{array}{|r|r|r|r|r|r|r|r|r|r|r|r|}
\hline \mathrm{P} & \mathrm{P} & \mathrm{P} & \mathrm{P} & \mathrm{M} & \mathrm{M} & \mathrm{M} & \mathrm{M} & \mathrm{M} & \mathrm{M} & \mathrm{M} & \mathrm{M} \\
1 & 2 & 4 & 8 & 3 & 5 & 6 & 7 & 9 & 10 & 11 & 12 \\
\hline
\end{array}
$$

Figure (2). Moving parity bit to left corner Messages bits in figure (2) are linier format that we know as one dimensional method. it is possible that the data by linier format store by matrix format and hamming code calculated in both dimension and place in corner of data. Two diminution data show in figure (3).

\begin{tabular}{|l|l|l|l|l|l|l|l|}
\hline M11 & M12 & M13 & M14 & M15 & M16 & M17 & M18 \\
\hline M21 & M22 & M23 & M24 & M25 & M26 & M27 & M28 \\
\hline
\end{tabular}

\begin{tabular}{|l|r|r|r|r|r|r|r|}
\hline M31 & M32 & M33 & M34 & M35 & M36 & M37 & M38 \\
\hline M41 & M42 & M43 & M44 & M45 & M46 & M47 & M48 \\
\hline M51 &. &. &. &. &. &. & M58 \\
\hline M61 &. &. &. &. &. &. & M68 \\
\hline M71 &. &. &. &. &. &. & M78 \\
\hline M81 & M82 & M83 & M84 & M85 & M86 & M87 & M88 \\
\hline
\end{tabular}

Figure (3). Two dimension message bits

By placing of parity bits in left corner and down corner of data after calculate of row and column parity figure (4) show this state. Parity bits are $\mathrm{Pi}$ and Qi in row and column.

Pi and Qi parity bits will be stored in memory and send with serial method. In this case if a row or column of data bits have errors then Pi and Qi parity bits only see a bit in column or row and one row or column bits of error will be corrected.

\begin{tabular}{|c|c|c|c|c|c|c|c|c|c|c|c|}
\hline P81 & P41 & P21 & P11 & M11 & M12 & M13 & M14 & M15 & M16 & M17 & M18 \\
\hline P82 & P42 & P22 & P12 & M21 & M22 & M23 & M24 & M25 & M26 & M27 & M28 \\
\hline P83 & P43 & P23 & P13 & M31 & M32 & M33 & M34 & M35 & M36 & M37 & M38 \\
\hline \multirow[t]{4}{*}{ P84 } & P44 & P24 & P14 & M41 & M42 & M43 & M44 & M45 & M46 & M47 & M48 \\
\hline & . & & . & M51 & & & . & . & . & . & M58 \\
\hline & . & . & . & M61 & . & . & . & . & . & . & M68 \\
\hline & & & & M71 & & & & & 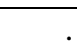 & & M78 \\
\hline \multirow[t]{5}{*}{ P88 } & P48 & P28 & P18 & M81 & M82 & M83 & M84 & M85 & M86 & M87 & M88 \\
\hline & & & & Q11 & Q12 & & & & . & 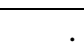 & Q18 \\
\hline & & & & Q21 & Q22 & & . & . & . & . & Q28 \\
\hline & & & & Q41 & Q42 & . & . & . & . & . & Q48 \\
\hline & & & & Q81 & Q82 & & & . & . & . & Q88 \\
\hline
\end{tabular}

Figure (4). Parity and message in two dimension mode

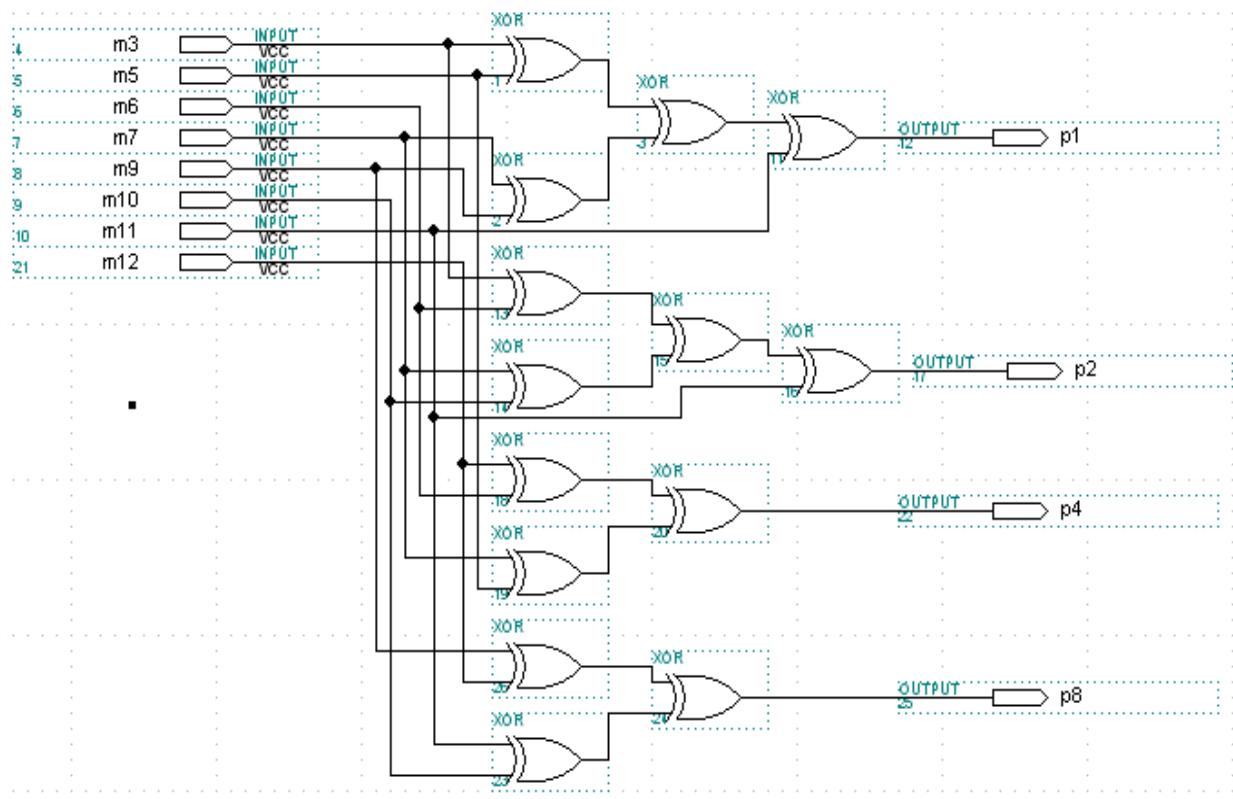

Figure (5). Parity bit generator circuit 


\section{International Journal of Innovative Research in Electrical, Electronics, Instrumentation and Control Engineering}

ISO 3297:2007 Certified

Vol. 5, Issue 2, February 2017

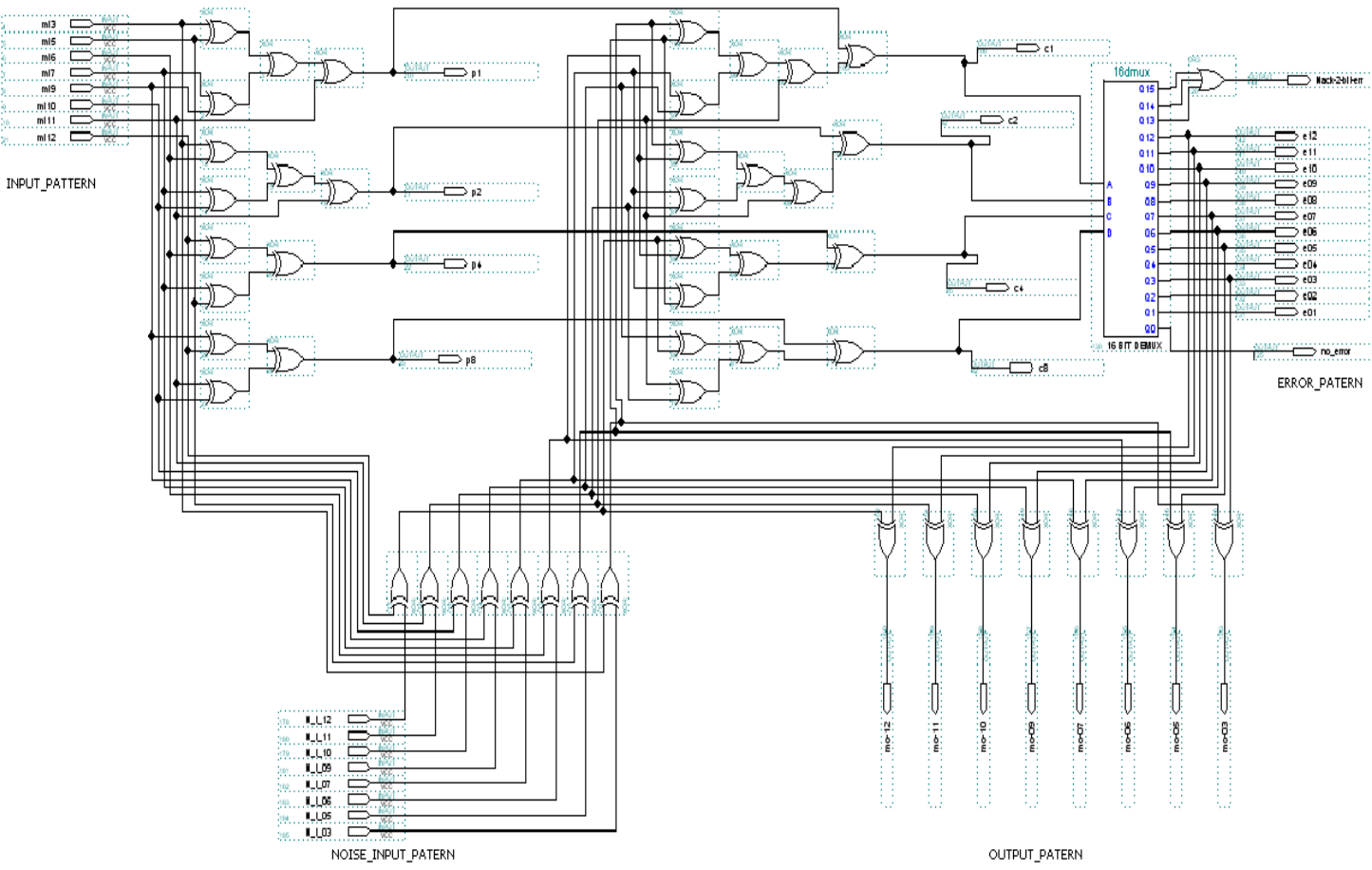

Figure (6). Error correction circuit with test circuit

If error bite have 2 or higher error bit in a both of row and column then it is not possible to correct this errors. In other diction if we have shadow error bits on both of row and column then it is not possible to correct errors and only can detecting those error bits. Based on equations from (1) to (4) the error correction and detection circuits are designed and shown in figures (5) and (6) that parity bits generated with XOR gates and store to register. The errors will be corrected if possible and if not possible the output of correction circuit of shown in figure (6) will activated and if corrected will be stored in register.

By showing this circuit with block diagram then show this circuit in row and column separately then figure (7) show this block diagram.

\section{IV.CONTROL OF POWER IN TWO DIMENSIONAL CIRCUIT DYNAMICALLY}

For decrease of power consumption must power off some party of circuit that shown in figure (7). It is possible that measurement the power level of power supply and manage correction and detection circuit and parity generator circuit in both of sender and receiver circuits. By decrease of power level of power supply the number of bits that can be corrected or detected and the power consumption will be decreased. This is done with the AND gated that shown in figure (7) beside the power manager block diagram.

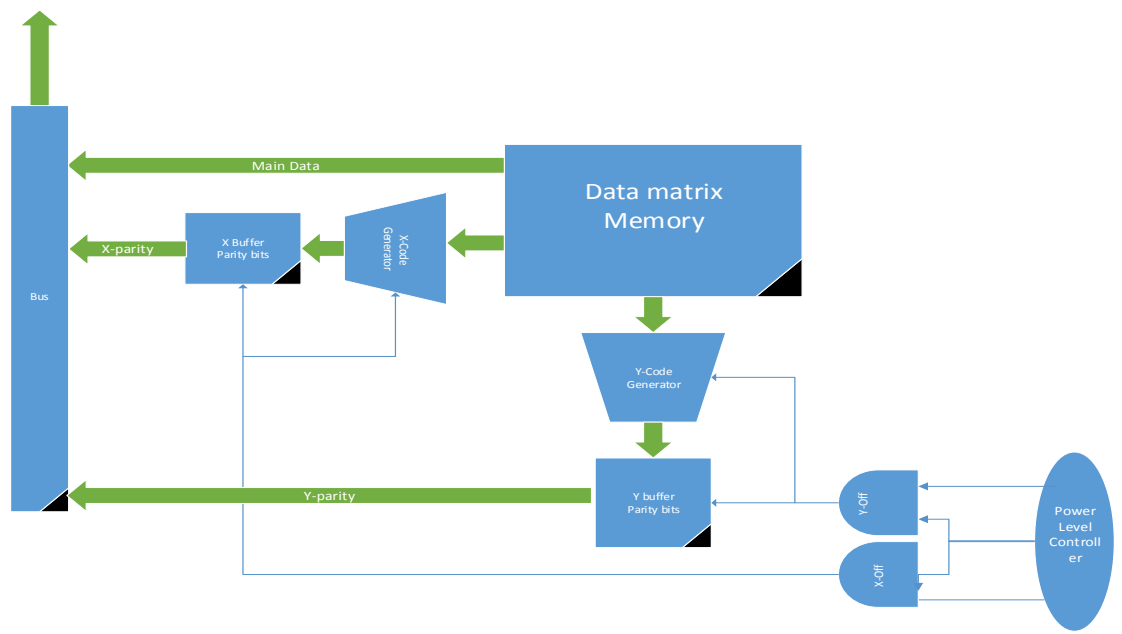

Figure (7). Two dimensional Error correction and detection block diagram in power aware state 


\section{International Journal of Innovative Research in Electrical, Electronics, Instrumentation and Control Engineering}

\section{ISO 3297:2007 Certified}

Vol. 5, Issue 2, February 2017

Both of sender and receiver are synchronous for power • detecting level and if one of the sender or receiver be decrease this power level then both of sender and receiver decrease the power consumption for doing same protocol in sender and receiver. For synchronous of sender and receiver we need two bit that separate state of those bits are introduce.

- (00) State: in this state the energy level is very low and error correction will not be done.

- (01) State: in this state the energy level is fair and error correction will be done in one dimension $\mathrm{X}$.
(10) State: in this state the energy level is good and error correction will be done in both dimension $\mathrm{X}$ and $\mathrm{Y}$. in this state maximum error correction will be done.

Therefore the state level selecting is done by equation (6). In this state we know that both of sender and receiver must select minimum state of power of each other for power aware done correctly.

Level Select

$=\operatorname{Min}\{$ Tx Level, $R x$ Level $\}$

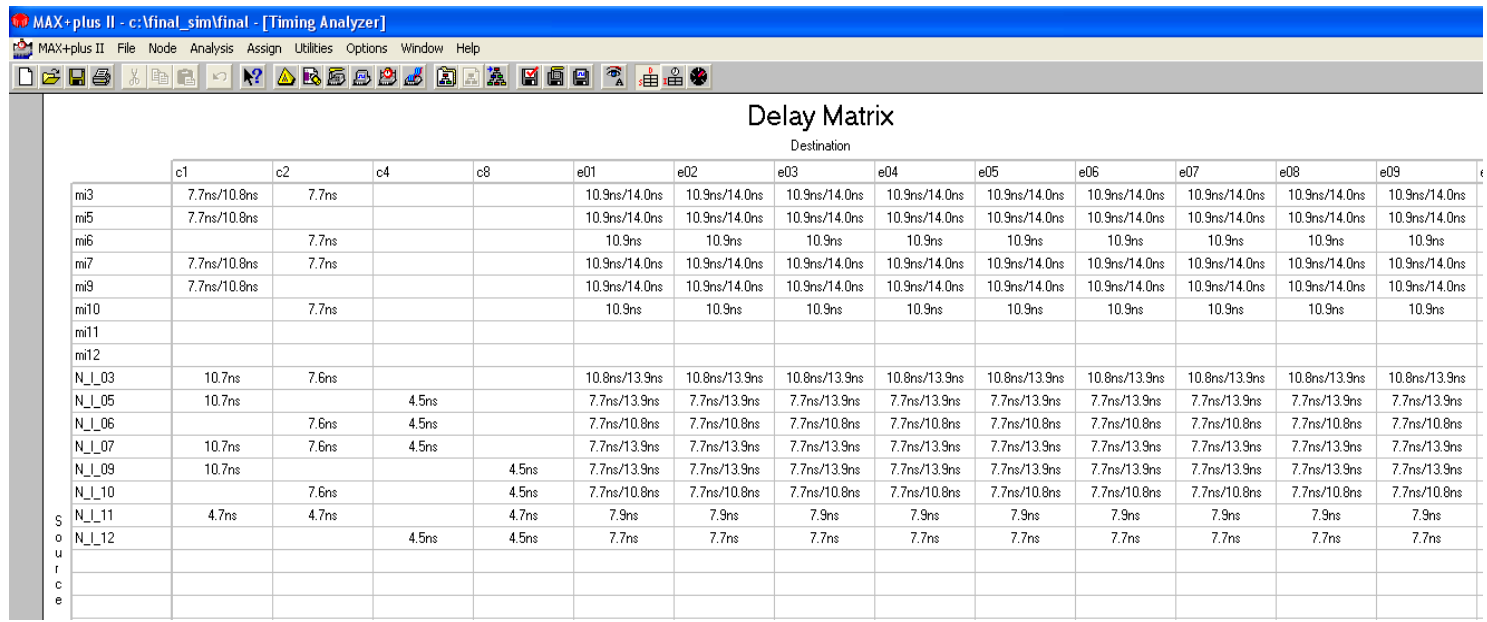

Figure (8). Simulator conclusion

In this state if only one of the senders energy level come down then receiver and other senders that connect to this receiver will work in level of minimum of the network. In other way we can say that the minimum energy of system is appointment by minimum energy of every sender or receiver of all connected systems.

Both of Tx or Rx level can be determined by (00), (01) or (10) that show the energy level of own system.

\section{SUMMARY AND CONCLUSIONS}

After simulating with maxplusII software for error detection and correction of above circuits and intern of error to system for test the result was shown in figure (8).

In Figure (8) we can see the errors that corrected after about 11 ns on e 08 column. Number of column are blank for wait to other dimension correct this error.

In the (00) state the system could not detect and correct the errors and all of column will be blank.

\section{REFERENCES}

[1] Prof W. Cary Huffman, Prof Versa Pless, Fundamentals of ErrorCorrecting Codes, Cambridge University Press, Published: (C) Cambridge University Press 2003, pp 2-3;

[2] Jeff Draper And Riaz Naseer, 2008, Parallel Double Error Correcting Code Design to Mitigate Multi-Bit Upsets in SRAMs,IEEE.
[3] Zhen Wang, Mark Karpovsky, Konrad J. Kulikowski, 2010, Design of Memories with Concurrent Error Detection And Correction by Nonlinear SEC-DED Codes, Received: 6 December 2009 / Accepted: 26 July 2010 / Published online: 11 August 2010 (C) Springer Science+Business Media, LLC 2010, pp 7-9.

[4] Gam D, Error-Detection Codes: Algorithms and Fast Implementation, IEEE Transactions ON Computers, VOL. 54, NO. 1, January 2005, pp 2-3

[5] Moris Mano, Digital Design, 1th Edition, Publication: Prentice Hall, 1984;FLEXChip Signal Processor (MC68175/D), Motorola, 1996.

[6] 6Eltayeb S. Abuelyaman And Abdul-Aziz S. Al-Sehibani, Optimization of the Hamming Code for Error Prone Media,2008, IJCSNS International Journal of Computer Science And Network Security, VOL.8 No.3, March 2008,pp 2-7.

[7] Eltayeb S. Abuelyaman, et al, 2008, Optimization of the Hamming Code for Error Prone Media, International Journal of Computer Science And Network Security, VOL.8 No.3, March 2008,pp 279283.

[8] K.V.Ganesh, D.Sri, Hari,M.Hema, Design And Synthesis of a Field Programmable CRC Circuit Architecture, International Journal of Engineering Research And Applications (IJERA), ISSN: 22489622 . 